\title{
IPFM: Intelligent Pressure Foot-Mouse
}

\author{
Hyun-Min Choi ${ }^{1}$, Yeong Seob Jeong ${ }^{1}$, Seung-Jun Son ${ }^{1}$, \\ Jae Gi Son ${ }^{2}$ and Seung Eun Lee ${ }^{1, *}$ \\ ${ }^{1}$ Dept. of Electronic Engineering \\ Seoul National University of Science and Technology, Seoul, Korea \\ ${ }^{2}$ Medical IT Convergence Research Center
}

Korea Electronics Technology Institute

\begin{abstract}
In this paper, we propose the mouse to control a computer with a shoe called IPFM: intelligent pressure foot-mouse. It is mainly composed of five parts including pressure sensor (FSR) embedded in a shoe, 3-axis acceleration sensor in front of shoe to measure a foot motion, computing module (FPGA), wireless communication module and visualization module (LABVIEW). From construction of the five parts, we developed a new input device called IPFM, which can be used by people who have difficult in using their hands to operate computers or devices easily. The Experimental results demonstrate the feasibility of our proposal.
\end{abstract}

Keywords: User Interface, foot mouse, computer system

\section{Introduction}

The development of the computer made human life living in comfortable circumstances. Most people usually spend time using computer nearly in half a day. Using computers become a part of our daily routine and people even use it for banking, shopping and telecommuting.

As the world is changing so fast, using computer becomes necessary to get a lot of information and knowledge efficiently. However, there are many people who suffer pain in their hands to operate computers. Our IPFM (Intelligent pressure foot-mouse) is designed to facilitate the computer especially for people who have difficulty in using their hands to operate devices. Our system is portable, wearable and compact. Thus, we expect that it helps the people who have a problem in their hands and makes efforts to promote their handicapped welfare.

Although it is designed for people with disabilities in hand, it just could not narrow it down to one field. It is useful to people who use a computer during many hours in a day. These people also suffer pain in their arm. It finally caused a new syndrome called "the mouse elbow" that people who like to play game suddenly suffer pain in the fingers and then massage their hand unwittingly. In this case, it can be considered as RSI [1]. Repetitive strain injury called RSI is an injury of the musculoskeletal and nervous systems that may be caused by repetitive tasks, forceful exertions, vibrations, mechanical compression pressing against hard surfaces, or sustained. The types of RSIs that affect computer users may include nonspecific arm pain or work related upper limb disorder. So there is a growing need to introduce

${ }^{*}$ Corresponding author, E-mail: seung.lee@seoultech.ac.kr, Tel: +82-2-970-9021 
alternative mouse in preparation for people who have difficult to use their hands to operate the devices.

In this paper, we propose the mouse to control a computer with a shoe called IPFM: intelligent pressure foot-mouse. Our IPFM system is also quieter and easier to handle than other foot devices. It is mainly composed of four parts including pressure sensor (FSR) embedded in a shoe, 3-axis acceleration sensor in front of shoe to measure a foot motion, computing module (FPGA) and wireless communication module. And IPFM work on the PC using LABVIEW. From construction of IPFM's five parts, we developed a new input device called IPFM, which can be used by people who have difficult in using their hands to operate computers or devices easily. In addition, our platform can be used in gait recognition. We were inspired by an FASH system [2]. It used pressure sensors embedded in a pair of shoes to detect the tiredness, and inform the user of incorrect value in order to show the degree of tiredness. Inspired by the applications of that system, we try to find the way to work the computer-mouse and the gait recognition together.

This paper is organized as follows. In Section 2, we introduce the related work and we described the construction of system in Section 3. In Section 4, we introduce function of each part of hardware. We then evaluate its validity and simplicity, by describing the operation of IPFM system in Section 5. Finally, our paper presents the future works in this area and concludes in Section 6.

\section{Related Works}

In recent years, researchers have focused on wearable computers and sensor device. From the miniaturization revolution in electronics, sensors and battery technologies, researchers was able to make the wearable alternative device that is light, compact, and convenient. For example, Kim [3] developed a mouse that is controlled by the movement of head and blink of an eye, which uses the gyro sensor and infrared sensor. Similarly there are many alternative mouse such as the track ball, joystick and keypad of keyboard [4]. In addition, our platform can be used in gait recognition. Vel'azquez [5] proposed to instruct the direction to user by using sensors attached to shoe. User can know the direction they want to go, as 16-point array of actuators have different vibration frequencies. So it is possible to use foot-tactile information for navigation in space. Minoru [6] developed a system for actualizing a new style of dance performance. It can make music using step when a dancer dances to the rhythm of the music. Baghebani [7] designed the mobile electronics that is a battery charging device using a rotary arm extending down from the sole. Because almost battery technology and device limited the size and weight, people could not use it in life time. But Baghebani eventually made the small electrical generator through their system of stepped-up gearbox. We investigated the possible application of shoe-based sensor interface and tried to extract the useful data about the information of motion when people took different actions such as working, standing and sitting. We proposed foot motion recognition system for potential use of mouse for human computer interaction and extend our work in this paper [8].

\section{System Overview}

A Shoe of the hardware structure is as follows (Figure 1): First, the five inputs coming from the FSR pressure sensor that are attached to the soles of shoes enter the amplifier. The amplified five analog signals and three axis acceleration sensor $\mathrm{x}, \mathrm{y}, \mathrm{z}$ inputs go into the external $\mathrm{AD}$ converter. In other words, a total of eight analog signals enter the external $\mathrm{A} / \mathrm{D}$ 


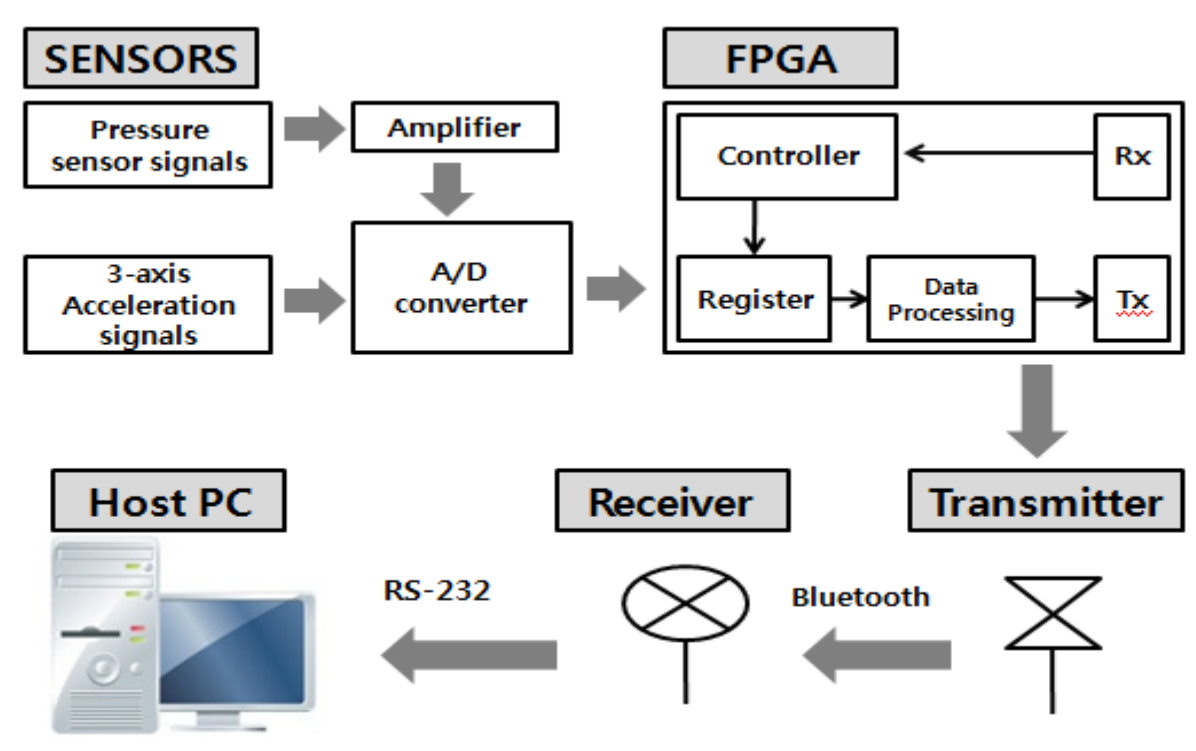

Figure 1. Block diagram of our IPFM system

converter and they are converted to 8 bit digital signals. After the 8 bit signals go into the FPGA, they are forwarded to a RS232 serial port at a speed of 9600bps. The data values are sent with Bluetooth communication and monitored on the Host PC. A Labview program in the Host PC provides the way for user to control the mouse using the foot. We implemented the control logic in FPGA, which enables the realization of our system in $\mathrm{SoC}$ in future as shown in Figure 2.

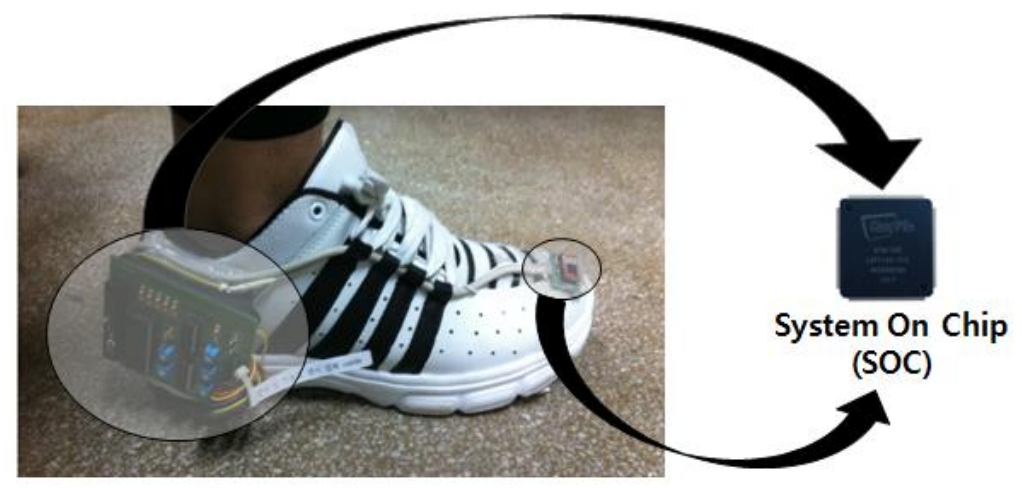

Figure 2. Photograph of our IPFM

\section{IPFM Hardware Architecture}

In this section, we describe the each function of hardware according to operating sequence of each function. IPFM hardware is composed eight of sensors, amplifier and ADC chip. Pressure sensors and a 3-axis accelerometer sensor are embedded in a sole. An amplifier and ADC chip are manufactured in a circuit board (See Figure 3). Also variable resistances are seen in Figure 4 which control voltage gains amplified by amplifier. 


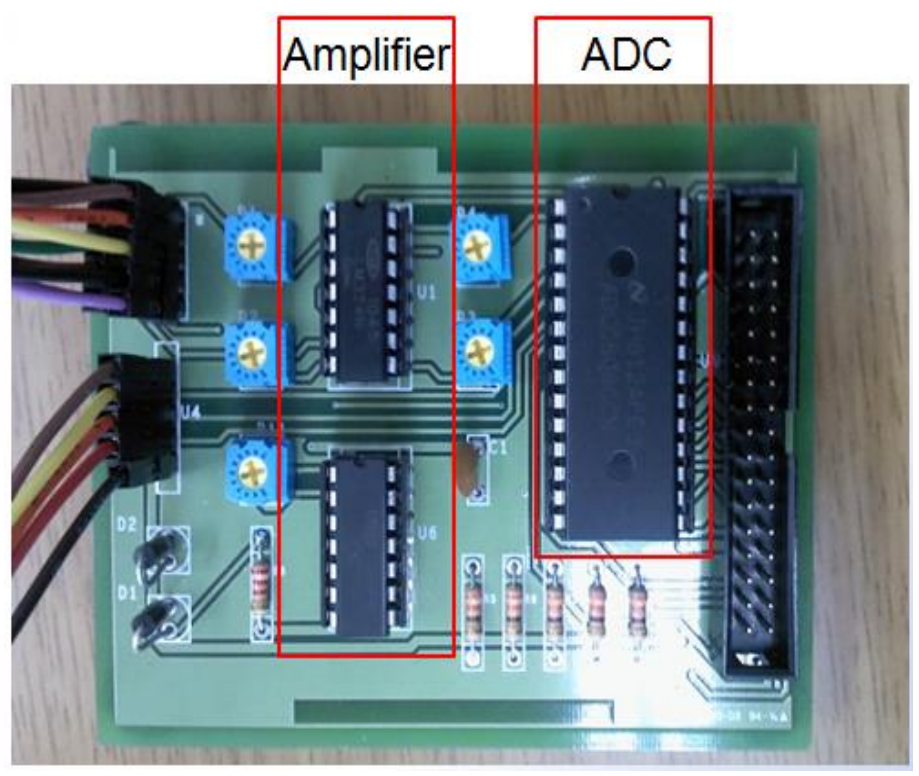

Figure 3. Photograph of the acquisition unit

\subsection{Hardware}

The eight of sensors are used in IPFM system. They consist of the five FSR pressure sensors and 3-axis accelerometer for $\mathrm{x}, \mathrm{y}, \mathrm{z}$ signals. Figure 5 shows that the FSR pressure sensors are placed according to position which receives a lot of the weight. The five FSR pressure sensors are going to enter the following amplifier circuit. The reason for using amplifier is that it can be possible to change the output voltage by changing resister. Amplified five analog signals and three-axis accelerometer $\mathrm{x}, \mathrm{y}, \mathrm{z}$ analog signals get into an external A/D Converter. The reason for using a separate A/D Converter is because general FPGA board does not have built-in ADC function.

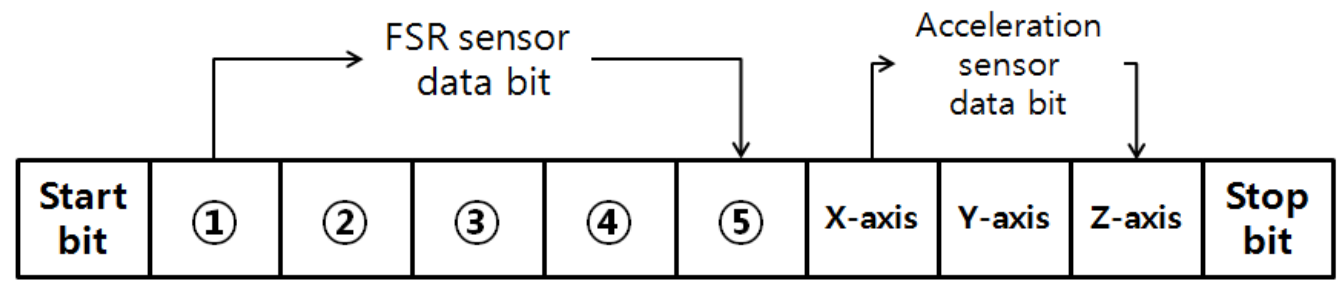

Figure 4. Packet format for communication

The control block is divided into an A/D Converter, a data processing, and a UART communication control section. The A/D Converter receives control signal and chooses one of eight analog signals per $300 \mu$ second and converts it to digital data. The RS232 serial communication (See Figure 4) transfers eight signals to the host PC at a speed of 9600bps. Firstly, it starts to send the start signal. Secondly, the pressure and acceleration sensor signals are transmitted sequentially. Finally, it sends the stop signal. Each of them is 1byte, so the 10byte signals are transmitted as a packet. 


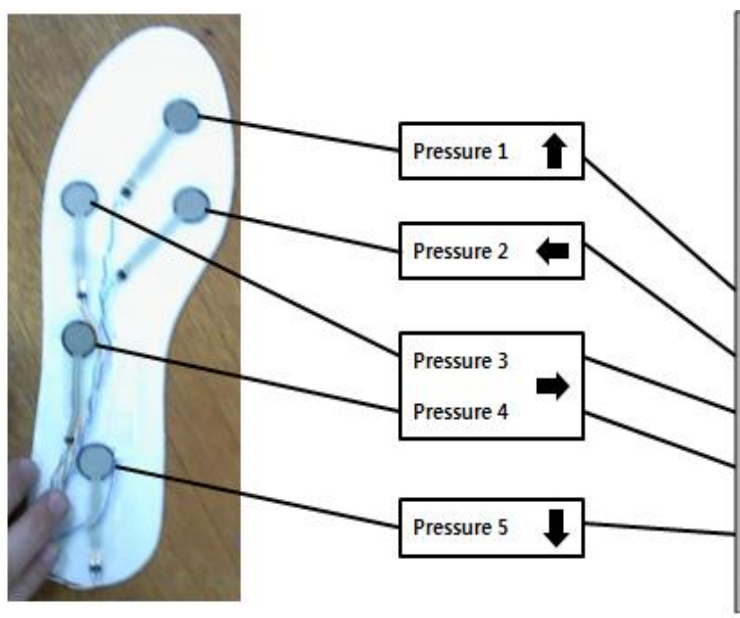

Figure 5. Location of pressure sensors

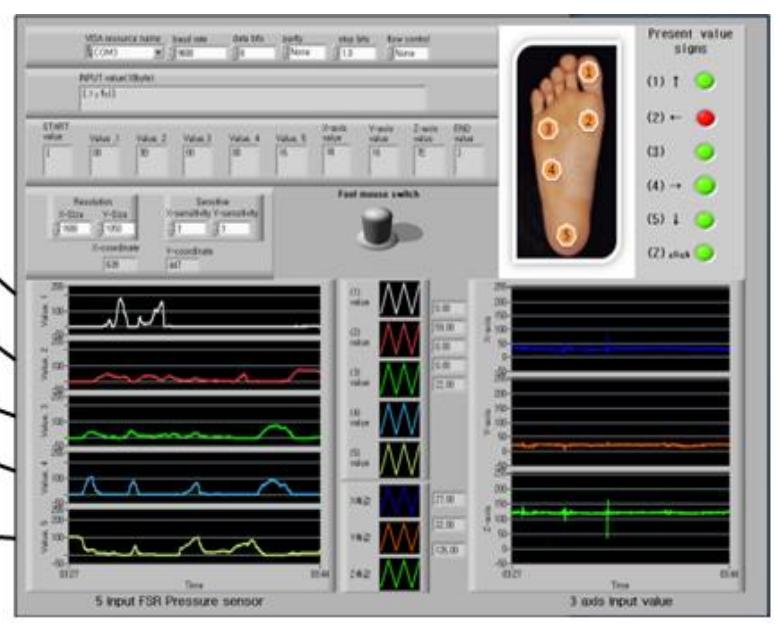

Figure 6. The Output signals of sensors using Lab view

\subsection{Host PC}

The data received through Bluetooth communication can be monitored by the Lab view program. Each signal can be verified with real-time graph (See Figure 6) which makes the users know which part of sensors are pressed and operate the motion of mouse cursor. Also the Lab view program makes it possible to save decimal data as a text or Excel files. Thus, the data stored in Excel could be used to investigate sensitivity change of IPFM to reduce a stress fracture of the foot in the future. We divide the motion of mouse into three classes: (1) mouse movement in two dimensions that is the $x-y$ position of mouse cursor on the screen; (2) single click of left button; (3) double click of left button. These three functions are usually used in computer work. When people wear the IPFM on the computer, they control the computer mouse without hands as pushing the each pressure sensor embedded in a shoe.

\section{Experimental Results}

In order to verify the functionality of our IPFM, we established the experimental environment which connects the IPFM to a Host PC. We extracted the received data and verified that the mouse cursor is rightly moved in accordance with the date value.

\subsection{Using FSR to produce motion of mouse cursor}

Positions of 5 force sensor embedded in a shoe were selected by experiment using molding technique. The force sensors are installed at the toe, left side of the sole, right side of the sole and the heel. When a user presses each of the force sensor located in sole, IPFM system enables the motion of the mouse-cursor and force intensity is shown in the real-time grape on the screen. Figure 7 displays a sample output waveform of the each force data when the user uses the IPFM system on computer. Each part of performance property in the IPFM is as in the following. If the part in the front of shoe is pressed, the IPFM makes the mouse-cursor go up. If the part on the left side of the shoe is pressed, the mouse-cursor will be moved to the left. The other performance property of going right and down are based on the same principle as above. Our system has absolutely no noises at all because our system does not need to drag the shoes. 


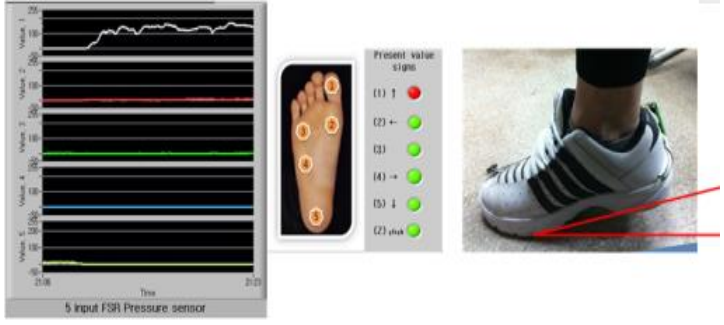

(1) Output signal of going up the mouse

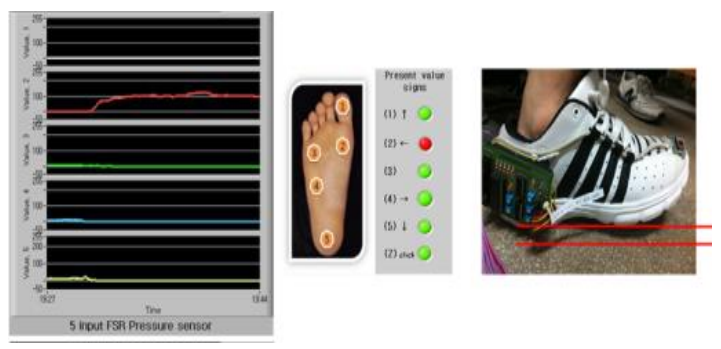

(3) Output signal of going right the mouse
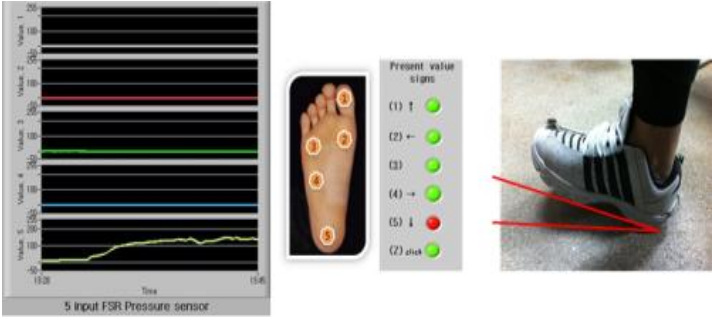

(2) Output signal of going down the mouse
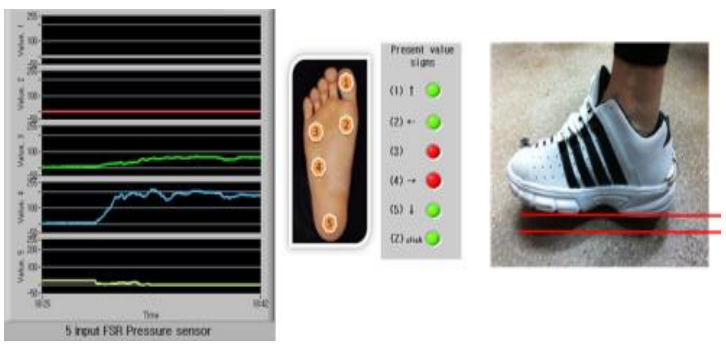

(4) Output signal of going right the mouse

Figure 7. The output waveform of each sensor measured, depending on each angle

Also we programmed the IPFM system only to work as the mouse when the IPFM system received data more than threshold value set. Thus, if user feels fatigue in their leg while using the IPFM, the threshold value can be adapted to suit individual conditions. For example, if users are so young or so old and they do not have the strength enough to press the sensor located in sole. Then, they can operate the foot-mouse with a wet finger by reducing the threshold value.

\subsection{Use accelerometer at the toe to produce single click of the left button}

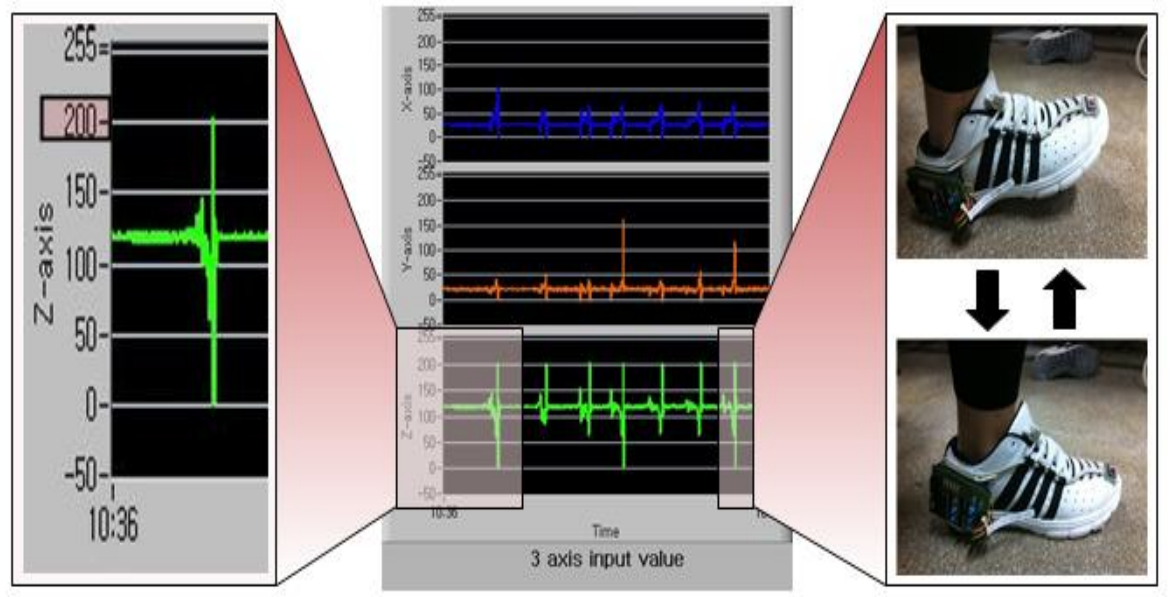

Figure 8. The output waveform of z-axis when user shook the shoe 
Accelerometer at the top of the shoe is adapted for a single click. The accelerometer sensor can output $\mathrm{x}$-axis acceleration, $\mathrm{y}$-axis acceleration and $\mathrm{z}$-axis acceleration. Among these outputs, we choose the z-axis acceleration for click function, because it reacted sensitively among the outputs of acceleration sensor when we shook the foot in the air and it was easy to control. Figure 8 displays a sample output wave form of z-axis acceleration when the shoe shook up and down. If the acceleration is bigger than the threshold value (200), the IPFM system has the click function of the left button.

\subsection{Setting the mouse cursor speed}

An output of the pressure sensor was affected by the weight of foot. The obtained outputs from the user's foot pressure sensors are directly connected to the cursor velocity. According to pressure intensity, the mouse cursor speed is changed. The outputs are represented by numerical value that is in the range of 1 to 255 . We divided this numerical value into three periods based on mouse cursor speed. Firstly, when the pressure values are between 1 and 80 , the mouse curser moved 2 pixels at a speed of $9600 \mathrm{bps}$. Secondly, the pressure values are between 81 and 160, the mouse curser moved 4 pixels at a speed of 9600 bps. Thirdly, if the pressure values are between 161 and 255, the mouse curser moved 6 pixels at a same rate.

\subsection{Effective Application}

Through using our system, it enables to apply to prevention of a RSI and assist people who have difficulty in using their hands to operate computer. Also it is useful in some kind of place such as scene of medical treatment and assembly line. For example, the doctor while undergoing surgery would be hard to use computer because they must wear glove storage. And workers having to use both hands on the assembly line are impossible to use computer in case of necessity. If IPFM is used to these situations, it can come in pretty handy. Furthermore, it can be also used to application for gait recognition. If the pressure data of healthy person are compared with the pressure data of patients by using the IPFM, people could tell at a glance what went wrong in their gait because the IPFM system charts their progress on a graph in real-time. So it could apply to field of foot rehabilitation or orthotherapy for athletes.

\section{Conclusions}

In this paper, we presented a wearable sensor shoes for a mouse that is operated without hands. The data from the shoe is transmitted to Host PC and make mouse move on the screen, establishing control channel between shoe and the computer.

Our system has advantage that does not make any noise even when the function of click takes effects. Also it is portable, light and easy to use due to its simple usage. Thus, it enables the IPFM to be used regardless location and age. Also this system is economical and suitable for everyday life because it embeds pressure sensors in a pair of shoes which allows users to wear them without any interference. If IPFM is realized in SoC, it will be more miniaturized.

There are a few concerns remain in the current design and implementation of IPFM. In this implementation, the cursor speed is actually designed to move just three level speeds at uniform velocity. So we are going to subdivide more classes of the cursor speed according to pressure intensity. Also we plan to actualize a mouse-drag functionality on our IPFM. 


\section{Acknowledgements}

This study was financially supported by Seoul National University of Science and Technology and Industrial Strategic technology development program(10035197) funded by the Ministry of Knowledge Economy(MKE, Korea).

\section{References}

[1] A. A. FaudZi and K. Suzumori, (Eds.), "Stiffness and Viscous Coefficient Characteristics for Ergonomics Chair Design", Proceedings of the IEEE/ASME Intelligent Mechatronics, (2011) July 3-7; Budapest.

[2] K. Yonekawa, T. Yonezawa, J. Nakazawa and H. Tokuda, (Eds.), "FASH: Detecting Tiredness of Walking People Using Pressure Sensors", Proceedings of 6th Annual International Congress of Mobile and Ubiquitous Systems: Networking and Services, MobiQuitous, (2009) July 13-16; Toronto, Canada.

[3] S. Kim, M. Park, S. Anumas and J. Yoo, "Head mouse system based on gyro and opto sensors", Proceedings of the 3rd International Conference on Biomedical Engineering and Informatics, (2010) October 16-18; Yantai.

[4] G. Savioz, M. Markovic and Y. Perriard, (Eds.), "Towards multi-finger haptic devices: A computer keyboard with adjustable force feedback", Proceedings of International Conference of Electrical Machines and Systems, (2011) August 20-23; Beijing, China.

[5] R. Vel'azquez, O. Baz'an and M. Magana, (Eds.), "A Shoe-Integrated Tactile Display for Directional Navigation", Proceedings of the IEEE/RSJ International Conference on Intelligent Robots and Systems, (2011) October 11-15; St. Louis USA.

[6] M. Fujimoto, N. Fujita, Y. Takegawa, T. Terada and M. Tsukamoto, "A Motion Recognition Method for a wearable Dancing Musical Instrument", Proceedings of the International Symposium on Wearable Computers, (2009) September 4-7; Linz, Australia.

[7] R. baghebani and M. Ashoorirad, (Eds.), "A power Generation System for Mobile Electronic Devices Using Human Walking Motion", Proceedings of the 2rd International Conference on Computer and Electrical Engineering, (2009) December 28-30; Dubai.

[8] H. Y. Choi, Y. S. Jeong, S. J. Son and S. E. Lee, "Foot Motion Recognition for Human-Computer Interaction", ICSIP 2012, (2012).

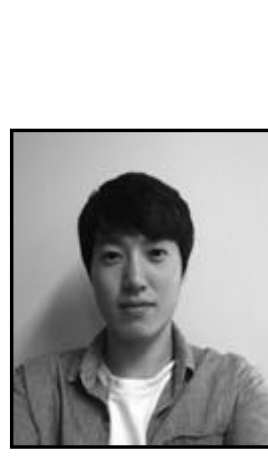

\section{Authors}

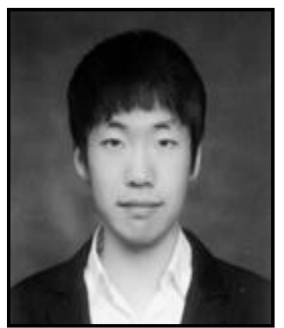

\section{Hyun-min Choi}

Hyun-min Choi is with UNIQUEST as an engineer. He has a B.S. degree in Electronic and Information Engineering from the Seoul National University of Science and Technology, Seoul, Korea. His research interest includes computer architecture and analysis language.

\section{Yeong-Seob Jeong}

Yeong-Seob Jeong is M.S. student in the Department of Electronic Engineering at Seoul National University of Science and Technology, Seoul, Korea. He has a B.S. degree in Electronic and Information Engineering from the Seoul National University of Science and Technology, Seoul, Korea. His research interest includes Computer Architecture, System-on-Chip, and Ubiquitous Computing and Fault tolerant System Design. . 


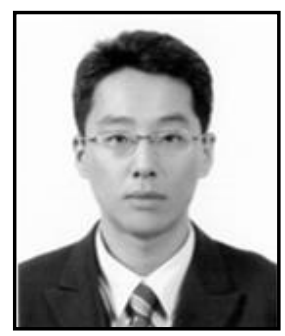

\section{Jae Gi Son}

Jae Gi Son is a Managerial Researcher in Medical IT Convergence Research Center at Korea Electronics Technology Institute (KETI), Sunggnam, Korea. He is currently studying for Ph.D. Degree in Computer Engineering from the Hankuk University of Foreign Studies, Korea. His research interest includes real-time system, information system, and medical system.

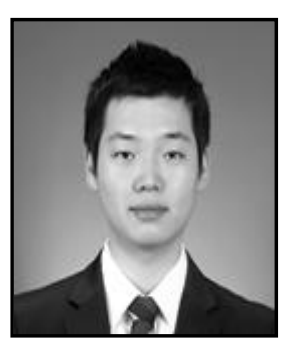

\section{Seung-Jun Son}

Seung-Jun Son is with ASML as an engineer. He has a B.S. degree in Electronic and Information Engineering from the Seoul National University of Science and Technology, Seoul, Korea. His research interest includes Circuit Designs and Image Processing.

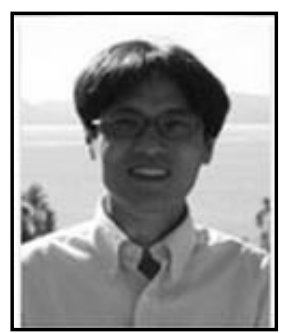

\section{Seung Eun Lee}

Seung Eun Lee is an Assistant Professor in the Dept. of Electronic and Information Engineering at Seoul National University of Science and Technology. He has a Ph.D. degree in Electrical and Computer Engineering from the University of California, Irvine and B.S. and M.S. degrees in Electrical Engineering from Korea Advanced Institute of Science and Technology (KAIST), Deajeon, Korea. His current research interests include computer architecture, multi-processor system-on-chip, low-power and resilient VLSI, and hardware acceleration for emerging applications. Prior to joining Seoul Tech in 2010, he spent 2 years as a platform architect at Intel Labs and 6 years as a researcher at Korea Electronics Technology Institute. 
International Journal of Multimedia and Ubiquitous Engineering Vol.8, No.5 (2013) 